\title{
QUASI-DETERMINISTIC MECHANICS OF THE HIGHEST WAVES \\ IN SEA STORMS
}

\author{
P. BOCCOTI \\ Professor of Civil Engineering \\ University of Reggio Calabria
}

LECTURE SPECIALE

\section{INTRODUCTION}

A person who observes a sea storm in an open sea, say from an offshore platform for a time-span of about half an hour, sees an irregular and nearly steady homogeneous wave field which is called "sea state".

The wave field is irregular because the waves at any fixed point have dimensions and shapes continuously variable in time, and at any fixed instant have dimensions and shapes different from one point to another. The wave field is nearly steady because the per cent variation of the energy content that wind is able to produce in half an hour within a sea storm is normally small. Finally, the wave field is nearly homogeneous because differences in the mean energy within the range of vision are typically negligible.

If the observation point, rather than in open sea, is near the coast, say from a wallbreakwater. what is seen is an irregular steady wave field being no longer homogeneous. Indeed the wave energy changes from one point to another due to the interaction with the structure. The phenomenon is that of reflectiondiffraction of a sea state.

A theory of sea states in an open sea was developed from the 50 s and 60s with important contributions by Longuet-Higgins (1963) and Phillips (1967). The first hypothesis is that a sea state is the sum of an infinitely large number $N$ of regular periodic waves with infinitesimal amplitudes, and with frequencies, directions and phases being different from one another. The relevant analytical form for the wave elevation and the velocity potential to Stoke's first order is

$$
\eta_{R}(x, y, t)=\sum_{i=1}^{N} \alpha_{i} \cos \left(k_{i} \sin \theta_{i} x+k_{i} \cos \theta_{i} y-\omega_{i} \dagger+\varepsilon_{i}\right)
$$




$$
\begin{gathered}
\phi_{R}(x, y, z, t)=g \sum_{i=1}^{N} \alpha_{i} \omega_{i}^{-1} \frac{\cosh k_{i}(h+z)}{\cosh _{i} h} \sin \left(k_{i} \sin \theta_{i} x+k_{i} \cos \theta_{i} y-\omega_{i} \dagger+\varepsilon_{i}\right), \\
k_{i} \tanh k_{i} h=\frac{\omega_{i}^{2}}{g}
\end{gathered}
$$

where $h$ is the bottom depth, $x, y$ are orthogonal axes in the horizontal plane, $z$ is the vertical upward axis with origin at the mean water level, and $\phi i$ is the angle that the propagation axis of the ith small wave makes with $y$-axis. The second hypothesis is that, for the same wind generation being repeated many times on the same basin, sea states all with the same frequency spectrum

$$
E(\omega) \delta \omega=\sum_{i} \frac{1}{2} \alpha_{4}^{2} \text { for } i \text { such that } \omega<\omega_{i}<\omega+\delta \omega
$$

and the same directional spectrum

$$
S(\omega, \theta) \delta \omega \delta \theta=\sum_{i} \frac{1}{2} \alpha_{i}^{2} \text { for } i \text { such that } \omega<\omega_{i}<\omega+\delta \omega \text { and } \theta<\theta_{i}<\theta+\delta \theta
$$

would be produced. Morever, the values of the phase angle $\epsilon_{i}$ would be distributed uniformly and purely at random in $(0,2 \pi)$.

On those assumptions wave elevation (1.a) and velocity potential (1.b) represent random stationary Gaussian processes of time.

\section{QUASI-DETERMINISM THEORY}

Provided to know that at a point $x_{0}$. yo within wave field (1). at a time instant to there is a wave with a height $H$ very large with respect to the mean $\left(H / \sqrt{m_{0}} \rightarrow \infty\right.$, $m_{0}=$ variance of $\left.\eta_{R}\right)$, the probability approaches 1 that the wave elevation is the sum of a deterministic component $\eta_{D}$ for order $\left(\frac{H}{\sqrt{m_{0}}}\right) \sqrt{m_{0}}$, plus a random component of order $\left(\frac{H}{\sqrt{m_{0}}}\right)^{0} \sqrt{m_{0}}$. The expression of $\eta_{0}$ is

$$
\begin{aligned}
& \eta_{D}\left(x_{0}+X_{,} y_{0}+Y, t_{0}+D\right)=\frac{H}{2}\left[<\eta_{R}\left(x_{0}, y_{0}, t\right) \eta_{R}\left(x_{0}+X_{,} y_{0}+Y, t+D\right)>+\right. \\
& \left.-<\eta_{R}\left(x_{0}, y_{0}, t+T^{*}\right) \eta_{R}\left(x_{0}+X_{,} y_{0}+Y, t+T\right)>\right] \Lambda\left[<\eta_{R}\left(x_{0}, y_{0}, t\right)>+\right. \\
& \left.-<\eta_{R}\left(x_{0}, y_{0}, t\right) m_{R}\left(x_{0}, y_{0}+Y, t+T^{*}\right)>\right]
\end{aligned}
$$

where to is the instant when the crest of the wave of given height $H$ forms at given point $x_{0}, y_{0}$, the angle brackets denote a mean with respect to time $t$

$$
\langle f(t)\rangle=\lim _{\tau \rightarrow \infty} \frac{1}{\tau} \int_{0}^{\tau} f(t) d t .
$$

and $T^{*}$ is the abscissa of the absolute minimum of the autocovariance function

$$
\psi(D)=\left\langle\eta_{R}\left(\mathrm{x}_{0}, \mathrm{y}_{0}, \mathrm{t}\right) \eta_{R}\left(\mathrm{x}_{0}, \mathrm{y}_{0}, \mathrm{t}+\mathrm{T}\right)\right\rangle
$$


Clearly, where deterministic component no approaches zero, say for large $X$ and/or $Y$ and/or $T$, the wave elevation approaches its random component, that is, it returns to be fully random.

Equally, the velocity potential is the sum of deterministic component

$$
\begin{aligned}
& \phi_{0}\left(x_{0}+X, y_{0}+Y, z, t_{0}+D=\frac{H}{2}\left[<\eta_{R}\left(x_{0}, y_{0}, t\right) \phi_{R}\left(x_{0}+X, y_{0}+Y, z, t+T\right)>+\right.\right. \\
& \left.-<\eta_{R}\left(x_{0}, y_{0}, t+T^{*}\right) \phi_{R}\left(x_{0}+X, y_{0}+Y, z, t+T\right)>\right] /\left[<\eta \eta_{R} \eta_{R}\left(x_{0}, y_{0}, t\right)>+\right](4, b) \\
& \left.-<\eta_{R}\left(x_{0}, y_{0}, t\right) \eta_{R}\left(x_{0}, y_{0}, t+T^{*}\right)>\right]
\end{aligned}
$$

which is of order $\left(\frac{H}{\sqrt{m_{0}}}\right) \sqrt{m_{0 \phi}}$ (mos being the variance of velocity potential $\phi_{R}$ ), and of a random component of order $\left(\frac{H}{\sqrt{m_{0}}}\right)^{0} \sqrt{m_{0}}$. Better to see the order of $\phi_{D}$. its eq. (4.b) should be divided and multiplied by $m_{0} \sqrt{m_{0 \phi}}$, with the consequence

$$
\begin{aligned}
& \phi_{0}\left(x_{0}+X, y_{0}+Y, z, t_{0}+T\right)=\frac{1}{2}\left(\frac{H}{\sqrt{m_{0}}}\right) \sqrt{m_{0}}\left\{<\eta_{R}\left(x_{0}, y_{0}, t\right) .\right. \\
& \tilde{\phi}_{R}\left(x_{0}+x_{,}, y_{0}+Y, z, t+D>-<\tilde{\eta}_{R}\left(x_{0}, y_{0}, t+T^{*}\right) \tilde{\phi}_{R}\left(x_{0}+x_{,} y_{0}+Y, z, t+T\right)>\right\} / \\
& /\left\{<\tilde{\eta}_{R}^{2}\left(x_{0}, y_{0}, t\right)>-<\tilde{\eta}_{R}\left(x_{0}, y_{0}, t\right) \tilde{\eta}_{R}\left(x_{0}, y_{0}, t+T^{*}\right)>\right\}
\end{aligned}
$$

where $\tilde{\eta}_{R}$ is the elevation related to its root mean square, and $\widetilde{\phi}_{R}$ is the velocity potential relates to its root mean square.

Result (4) was introduced by the author (1984-1989). It is based on a set of theorems holding for the wave fields where both the free surface elevation and the velocity potential are stationary random Gaussian processes of time at any point. Thus the result is not confined to the classic sea state (1) in an open sea.

In particular, if we place a vertical reflecting wall along axis $y=0$, the random wave field (1) is modified and takes the form.

$$
\begin{aligned}
& \eta_{R}(x, y, t)=2 \sum_{i=1}^{N} a_{i} \cos \left(k_{i} \sin \theta_{i} x-\omega_{i} \dagger+\varepsilon_{i}\right) \cos \left(k_{i} \cos \theta_{i} y\right) \\
& \Phi_{R}(x, y, z, t)=2 g \sum_{i=1}^{N} a_{i} w_{i}^{i} \frac{\cosh \left[k_{i}(h+z)\right]}{\cosh k_{i} h} \sin \left(k_{i} \sin \theta_{i} x-\omega_{i} \dagger+\varepsilon_{i}\right) \\
& . \cos \left(k_{i} \cos \theta_{i} y\right)
\end{aligned}
$$

where, under the hypotheses of sect. $1 \quad\left(N \rightarrow \infty, \varepsilon_{i}\right.$ distributed purely at random in $0.2 \pi$ and $\omega_{i} \neq \omega_{j}$ if $i \neq j$, both $\eta \frac{}{R}$ and $\phi_{R}$ represent stationary Gaussian processes of time at any point.

Another example is got if we place a semi-infinite vertical reflecting wall along line $y=0$. Then the random wave field (1) takes on the form 


$$
\begin{gathered}
\eta_{R}(r, \alpha, t)=\sum_{i=1}^{N} \alpha_{i}\left[F\left(r, \alpha ; \omega_{i}, \theta_{i}\right) \cos \left(\omega_{i} \dagger+\epsilon_{i}\right)+G\left(r, \alpha ; \omega_{i}, \theta_{i}\right) \sin \left(\omega_{i} \dagger+\varepsilon_{i}\right]\right. \\
\phi_{R}(r, \alpha, z, \dagger)=g \sum_{i=1}^{N} \alpha_{i} \omega_{i}^{-1} \frac{\cosh \left[k_{i}(h+z)\right]}{\cosh k_{i} h}\left[\left(G\left(r, \alpha ; \omega_{i}, \theta_{i}\right) \cos \left(\omega_{i} \dagger+\varepsilon_{i}\right)+\right.\right. \\
-F\left(r, \alpha, \omega_{i}, \theta_{i}\right) \sin \left(\omega_{i} \dagger+\varepsilon_{i}\right)
\end{gathered}
$$

where

$$
\begin{aligned}
& F(r, \alpha ; \omega, \theta)=A\left(u_{1}\right) \cos q_{1}+A\left(u_{2}\right) \cos q_{2}-B\left(u_{1}\right) \sin q_{1}-B\left(u_{2}\right) \sin q_{2} \\
& G(r, \alpha ; \omega, \theta)=A\left(u_{1}\right) \sin q_{1}+A\left(u_{2}\right) \sin q_{2}+B\left(u_{1}\right) \cos q_{1}+B\left(u_{2}\right) \cos q_{2} \\
& A(u)=\frac{1}{2}\left[1+\int_{0}^{u} \cos \left(\frac{\pi}{2} x^{2}\right)+\sin \left(\frac{\pi}{2} x^{2}\right) d x\right] \\
& u_{1}=2 \sqrt{\frac{k r}{\pi}} \sin \left[\frac{1}{2}\left(\alpha-\theta^{\prime}\right)\right], u_{2}=-2 \sqrt{\frac{k r}{\pi}} \sin \left[\frac{1}{2}\left(\alpha-\theta^{\prime}\right)\right], q_{1}=k r \cos \left(\alpha-\theta^{\prime}\right) \\
& q_{2}=k r \cos \left(\alpha+\theta^{\prime}\right)
\end{aligned}
$$

and $r$ and $\alpha$ are the polar coordinates with the origin at the wall-end, $\theta$ as usual is the angle of the wave direction with the $y$-axis and $\theta^{\prime}$ is equal to $\frac{\pi}{2}-\theta$.

Also eqs. (7) are exact to Stokes' first order and are based on the solution by Penny \& Price (1952) for the diffraction of the regular periodic waves. Here too. both $\eta_{R}$ and $\phi_{R}$ represent stationary Gaussian processes of time under the usual hypotheses, and thus result (4) applies also to this random wave field (Boccotti, 1988).

It is possible to verify that, provided random wave elevation $\eta_{R}$ and velocity potential $\phi R$ satisfy the wave differential equations to Stoke's first order as well as a set of boundary conditions also deterministic wave elevation $\eta_{D}$ and velocity potential $\phi D-e q \cdot(4)$ - satisfy those equations and boundary conditions.

Here it should be noted that the hypothesis of very large wave height $\mathrm{H}$ does not conflict with the Stokes hypothesis of very small wave height. Indeed. On the one hand, wave height $H$ has to be large with respect to the mean wave height, and on the other, it has to be small with respect to the wave length and the bottom depth.

The calculation of deterministic waves (4) can be done once the spectrum of the sea state is given. Indeed the averages in eq.(4) of no and $\phi D$ are related to spectrum $\mathrm{S}(\omega, \theta)$. In the case of sea states interacting with walls, the averages in eq. (4) prove to be related to the usual directional spectrum $s(\omega, \theta)$ of the waves that there would be if the wall was not there. As an example let us deduce the equation for the first average <.> in eq. (4a) in the case that the sea state interacts with an infinitely long wall. From eq. $(6 a)$ of the random wave elevation. we can write 


$$
\begin{aligned}
& <\eta_{R}\left(x_{0}, y_{0}, t\right) \eta_{R}\left(x_{0}+X, y_{0}+Y, t+T\right)>= \\
& =4 \lim _{\tau \rightarrow \infty} \frac{1}{\tau} \int_{0}^{\tau} \sum_{j=1}^{N} \sum_{j=1}^{N} \alpha_{i} \cos \left(k_{i} \cos \theta_{i} y_{0}\right) a_{j} \cos \left[k_{j} \cos \theta_{j}\left(y_{0}+Y\right)\right] \\
& . \cos \left(k_{i} \sin \theta_{j} x-\omega_{i}^{\dagger}+\epsilon_{D} \cos \left[\left(k_{j} \sin \theta_{j} x-\omega_{j} \dagger+\epsilon_{j}\right)+\left(k_{j} \sin \theta_{j} X-\omega_{j} T\right)\right] d t\right.
\end{aligned}
$$

and then putting the last cosine in the form cos.cos-sin.sin, inverting the order integral-sum and recalling that $\omega_{i} \neq \omega_{j}$ if $i \neq j$, we get

$$
\begin{aligned}
& <\eta_{R}\left(x_{0}, y_{0}, t\right) \eta_{R}\left(x_{0}+X, y_{0}+Y, t+T\right)>= \\
& =4 \sum_{i=1}^{N} \frac{1}{2} \alpha_{i}^{2} \cos \left(k_{i} \cos \theta_{i} y_{0}\right) \cos \left[k \cos \theta_{i}\left(y_{0}+Y\right)\right] \cos \left(k_{i} \sin \theta_{i} X-\omega_{i} D=\right. \\
& =4 \int_{0}^{\infty} \int_{\pi}^{\pi} S(\omega, \theta) \cos \left(k \cos \theta y_{0}\right) \cos \left[k \cos \theta\left(y_{0}+Y\right)\right] \cos (k \sin \theta X-\omega D) d \theta d \omega
\end{aligned}
$$

Where the second equality proceeds directly from the definition of spectrum eq.(3)-. The integral can be solved numerically, and the calculation proves to be expedite because of the quick convergence of the integrals, so that it can be quite successfully done by means of personal computers.

\section{A FEW PREDICTIONS OF THE QUASI-DETERMINISM THEORY}

\section{1- How g height wave forms in an open sea}

Eq. ( $4 a$ ) shows that the wave of given very large height $H$ at given point $x_{0}$. yo within sea stat (1) on a open sea, forms because of the transit of a wave group Fig. 1-. 


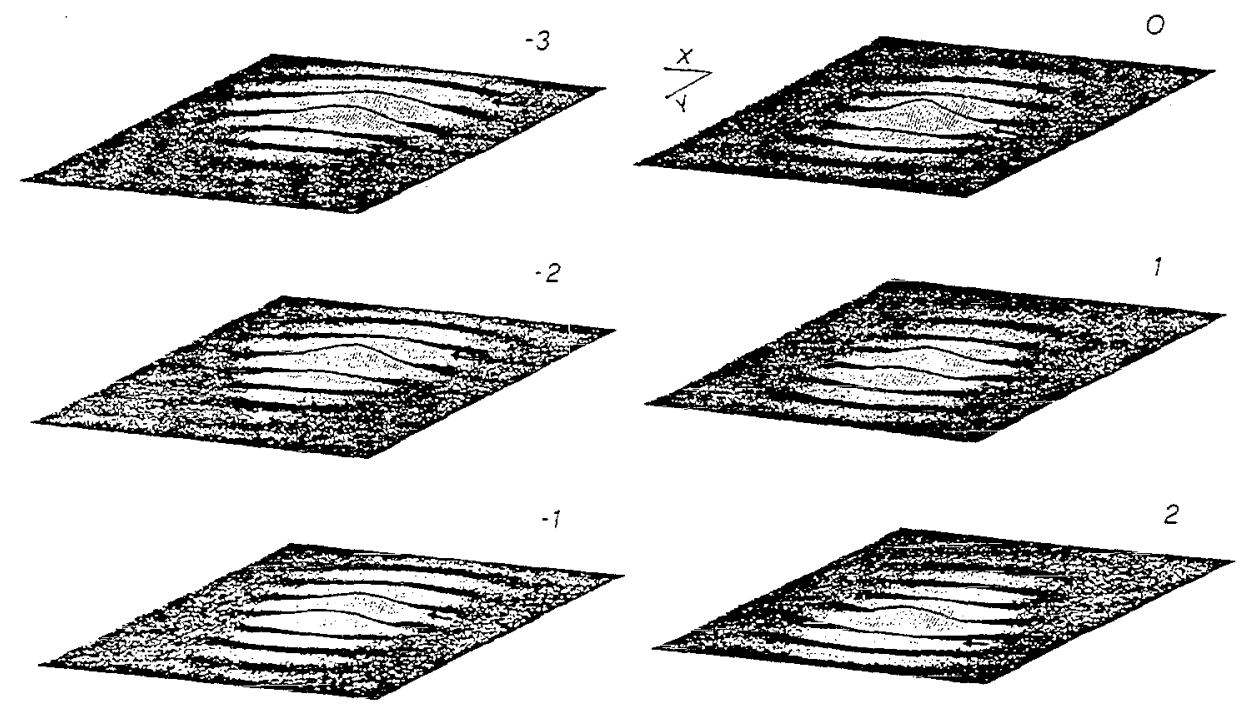

Fig. 1 : Typical mode of formation, within a random sea state, of a wave of given very large height $H$ at point $x_{0}, y_{0}$ in the center of the framed area. The waves are in an open sea on deep water and the framed area is 6 wave lenghts $L_{d}$ along $x$ axis per 8 La along $y$-axis. We see that the wave of the given very large height forms because of the transit of a well precise wave group.

The group has a development stage during which both the envelope and the wave front narrow till a minimum. Then a decay stage follows, with the opposite features. Each single wave having a celerity greater than the group runs along the envelope from the tail where it is born to the head where it goes to die (in the pictures a single wave is followed by an arrow during its evolution). Because of this phenomenon, single waves experience some really big transformations which have been recently confirmed by an experiment at sea, whose results will be shown at the 23 rd Conference on Coastal Engineering.

The wave of given very large height $H$ proves to be that at the center of the group at the apex of its development stage. Thus, theory in poor words says: if you record a wave a height $H$ very large with respect to the mean, the probability is very high that it is the central wave of a well defined wave group at the apex of its development stage.

The wave group of Fig. 3 is on deep water and it has been calculated from a characteristic spectrum that is the mean JONSWAP frequency spectrum 
(Hasselmann \& al. 1973) with $\cos 2 n\left(\theta-\theta_{0}\right)$ as spreading direction function ; $n$ is taken equal to 5 that is a characteristic value, to judge on data of storm seas (Forristall \& al, 1980). Here it should be remarked that, if we assume another wind wave spectrum, only a few details change but the essential features of the wave group mechanics do not change. in particular if the bandwidth grows, e.g. On passing from the JONSWAP to the Pierson \& Moskowitz spectrum, only the envelope narrows; and if the directional spread grows, e.g. on reducing the value of $n$, Only the width of the wave front reduces.

As to $\theta_{0}$, the angle that the dominant direction of the spectrum makes with the $y$-axis, it has been assumed to be zero, and we see that also the wave group moves along the $y$-axis. This means that a very high wave, with a very high probability, belongs to a group which moves along the dominant direction of the spectrum.

\subsection{How a high wave forms gt the wall}

The question is 'how does a wave with a given very large height $H$ form at a point $x_{0}, y_{0}$ in contact with the wall ?' and Fig. 2 is the answer given by eq.(4).

The wall is along the upper $x$-parallel side of the framed area and given point $x_{0}, y_{0}$ is at the center of the wall. The waves now are observed from offshore. The spectrum is the same as for the foregoing pictures, and its dominant direction makes a $20^{\circ}$ angle with the wall-orthogonal : $\theta_{0}=20^{\circ}$. The water is deep.
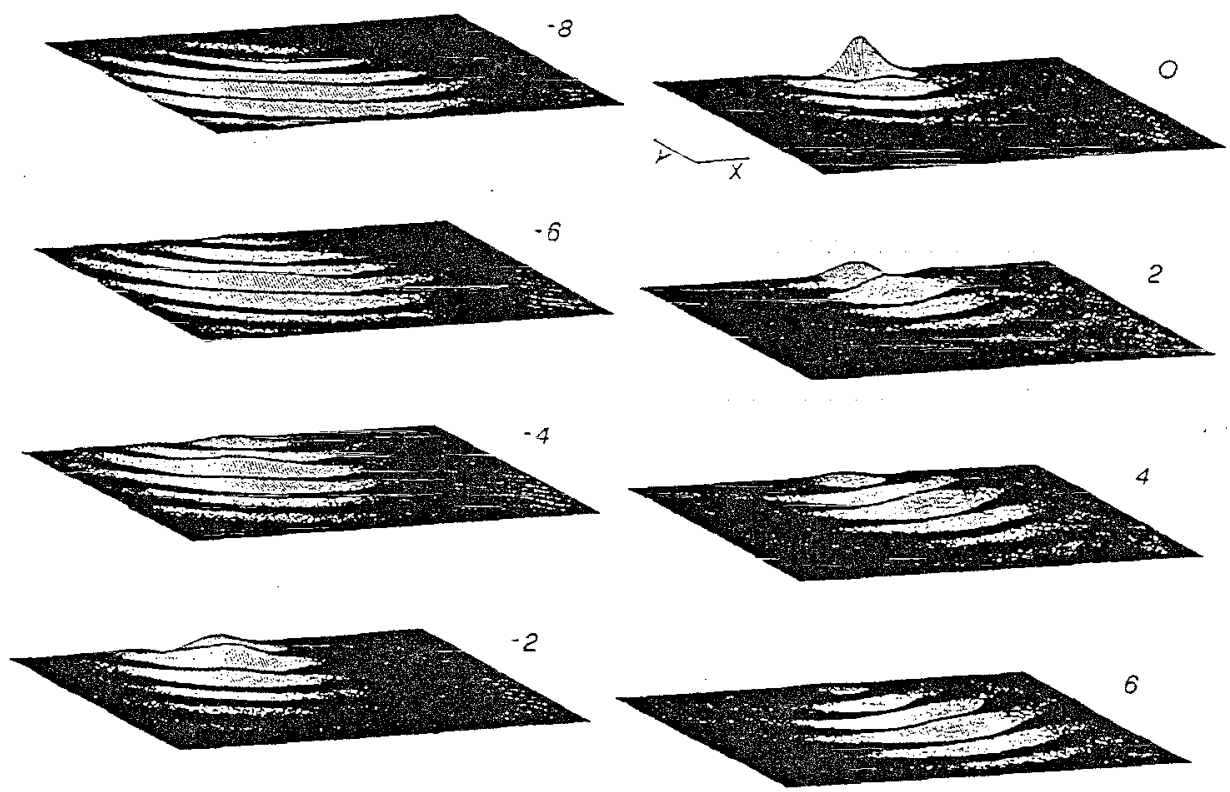

Fig.2 : Typical mode of formation of a wave of given very large height $H$ at the breakwater. The wall is along the upper $x$-parallel side and point $x_{0}$, yo of the wave of given height $H$ is at the wall center. The framed area is 8 wave lengths $L d$ along $x$-axis per 6 Lo along $y$-axis, the dominant direction of the sea state makes a $20^{\circ}$ angle with the walt-orthogonal; as a consequence we see the wave group 
that approaches the wall from the left and then is reflected mirrowise.

The first four pictures taken at regular intervals of $2 T_{d} T_{d}=$ peak period of the spectrum) from each other show a wave group approaching the wall. At time instant to ( $O$ in the picture) the wave of the given very large height $H$ is forming at the wall. It is the result of the reflection of the central wave of the group. Then, in the last three pictures, the wave group being reflected mirrorwise goes back seaward.

It will have been noted that, mean while the wave group is approaching the wall both the envelope and wave front narrow, and the reverse is the case when the group goes back. This reveals that the group reaches the wall at the apex of its develoment stage when its central wave obtains its maximum height. Thus the answer of the theory, in words, is'a very high wave at the wall, with a very high probability, takes place because a we!l defined wave group hits the wall when it is at the apex of its developement'.

\subsection{How a high wave forms off the wall}

Now the question is the'how does a wave with a given very large height $H$ form at a point $x_{0}$, yo two wave lenghts before the wall ?', and Fig. 3 is the answer given by eq. (4). This time, dominant direction of the spectrum is wall-orthogonal.
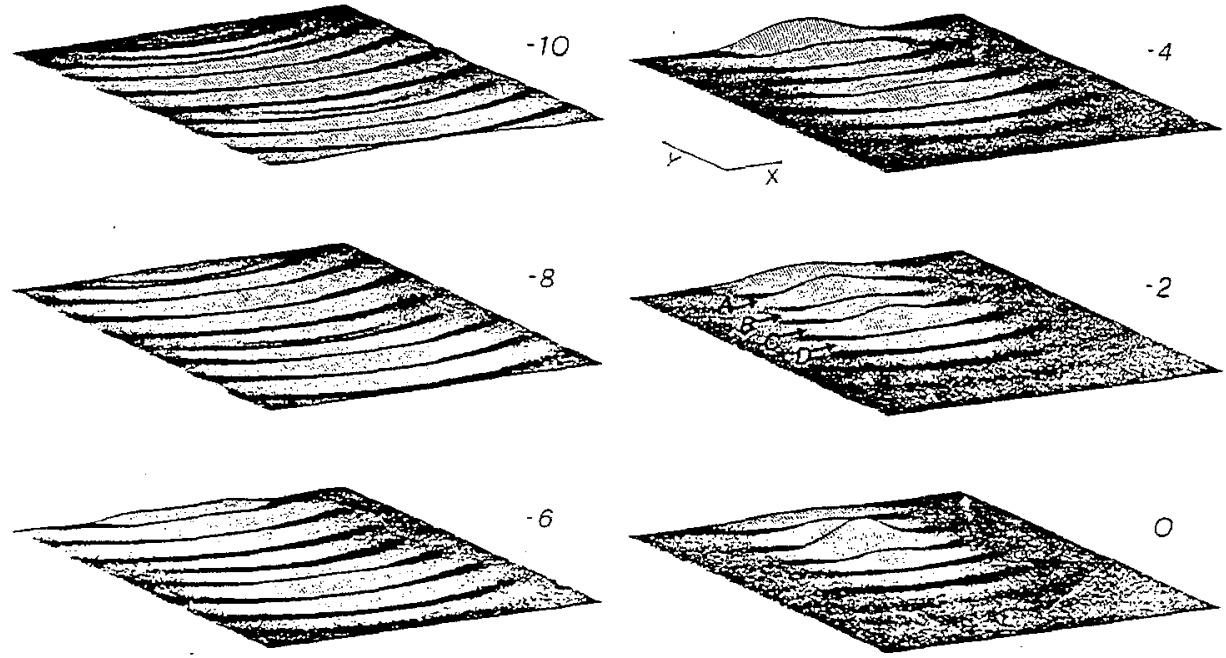

Fig. 3: Typical mode of formation of a wave of given very large height $\mathrm{H}$ at a point xo. yo two wave lengths far off a breakwater. We see two wave groups that approach the wall : the first group after having being reflected collides the second group that approaches the wall (the particle velocity before of the collision is shown in Fig.4). The wave of given very large height $H$ forms where the central waves of the two groups overlap. 
At instant to - 10Td two groups are distinguishable: the center of the first is nearly $3 L_{d}\left(L_{d}=\frac{G T_{d}^{2}}{2 \pi}\right)$ off the wall, the center of the second group is nearly $7 L_{d}$ from the wall. The tail of the second group is outside of the frame. The two groups approaching the wall appear more distinctly in the next two pictures at instants to $-8 T_{d}$ and to $-6 T_{d}$. The fourth picture, time instant to $+4 T_{d}$, shows the central wave of the first group being reflected by the wall. The group at that time is still building up so that the wave height at the wall is not so large as in Fig.2.

The fifth picture, time instant to-2Td, shows a complex scene: at the wall, the rear wave of the first group is being reflected, the next wave $(A)$ is the central wave of the first group which is regaining the sea: wave $B$ is a standing wave generated by the head wave of the first group advancing seaward and by the head wave of the second group advancing landward: wave $C$ is progressive like wave A but travels in the opposite direction: it is the central wave of the second group. which advances landward: finally wave $D$ is the rear wave of the second group. which likewise travels towards the breakwater. Fig.4 provides a readily understood confirmation of the nature of waves $A, B, C$ and $D$. Indeed it shows the horizontal component of the particle velocity calculated from the velocity potential -eq. (4b)-. It will be seen that under wave crest $A$, the horizontal velocity $v_{y}$ is negative, that is seaward oriented: under wave crest $B$, $v_{y}$ is practically zero, which reveals a peculiar characteristic of the standing waves: under wave crests $C$ and $D$. vy is positive, that is landward oriented. In conclusion, the scene at time instant to - $2 T_{d}$ is that of two wave groups approaching collision.

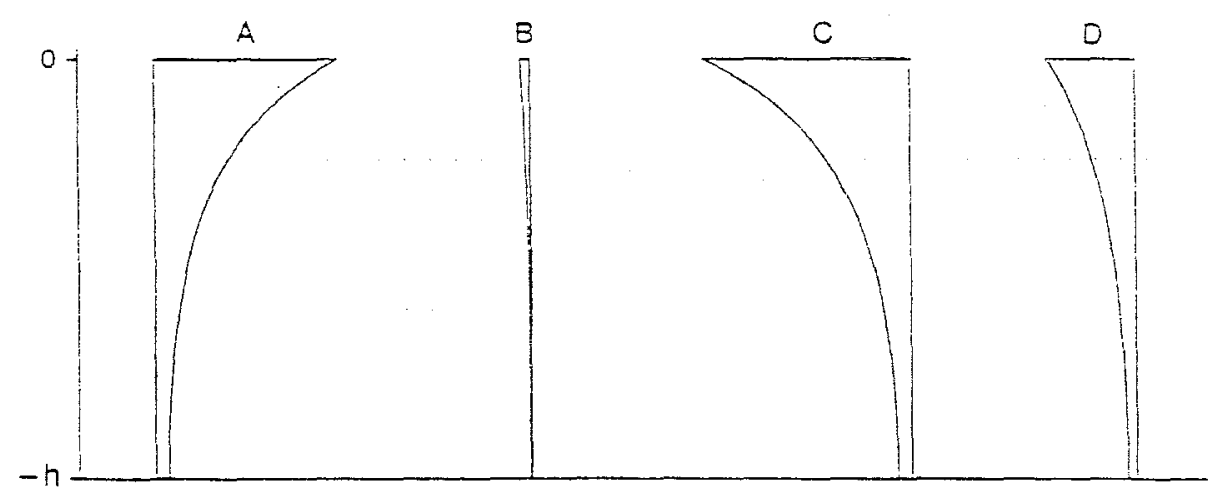

Fig. 4 : Horizontal particle velocity below the crests of waves A, B, C. D in Fig. 3 at instant to $-2 T$ d.

Two wave periods later, at time instant to ( $O$ in the picture), the collision is at its climax. The two central waves are overlapping and the wave of the given very large height $H$ is forming at given point $x_{0}, y_{0}$, it is the point at the center of the 
wave front below the maximum crest. What is shown at time instant to is an offshore standing wave field being generated by two opposing wave groups. Indeed, not only the central waves of the two groups are overlapping, but also the head wave of the first group and the rear wave of the second, and the rear wave of the first group and the head wave of the second are overlapping.
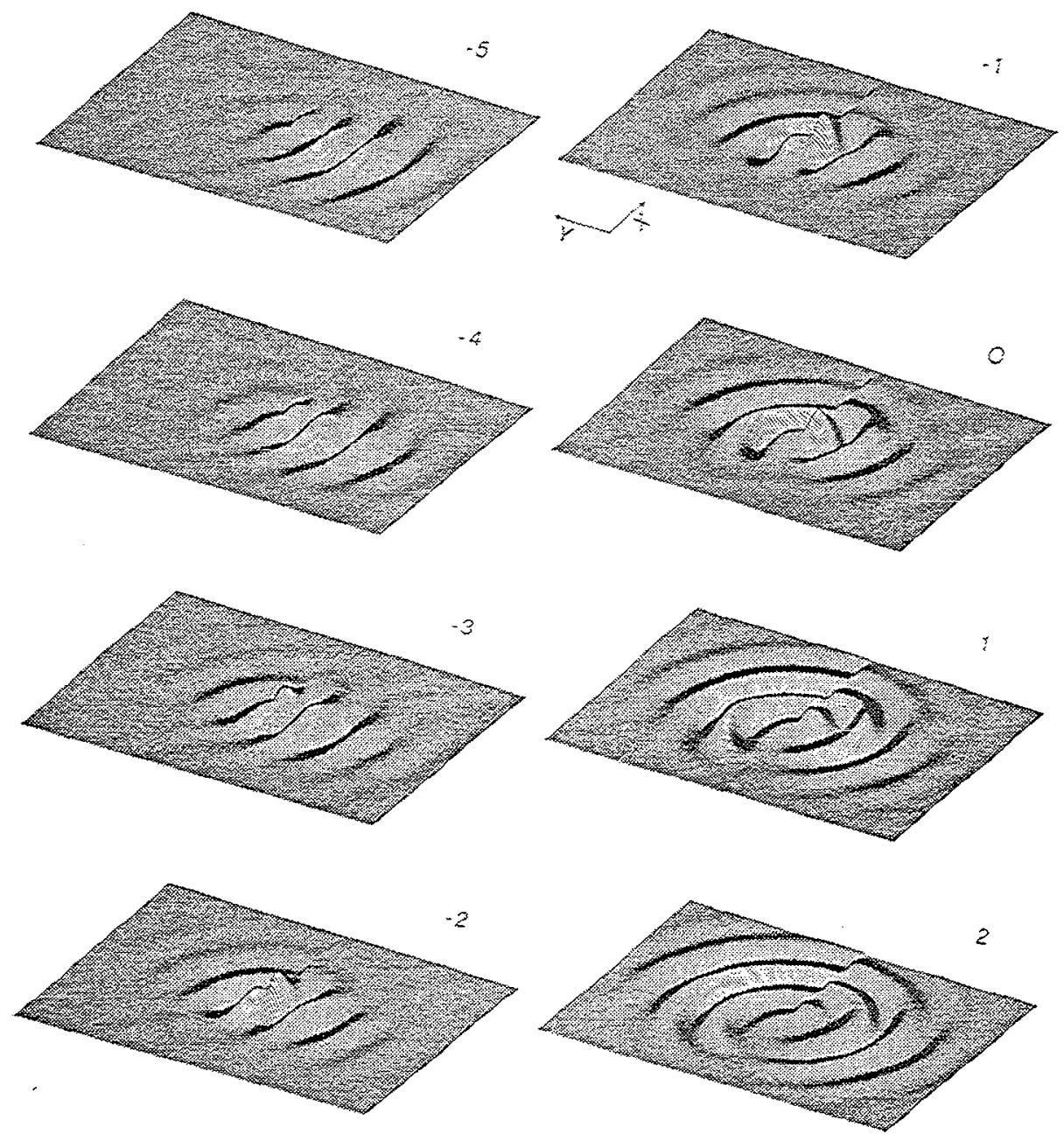

Fig.5: Typical mode of formation of a wave of given very large height $\mathrm{H}$ at a point in contact with the inward face of the wall, I wave length from the wall-end (the wall is parallel to $x$-axis and the wall-end is at the center of the framed area). The given height $\mathrm{H}$ is meant to be very large with respect of the mean wave height at the given point behind the wall. We see a wave groupe whose front-center impacts the wall-end.

\subsection{How a high wave forms in the shadow cone behind a wall}

Here the question is' how does it form a wave with a given very large height $H$ at a point in contact with the inward face of the wall : $r_{0}=L_{d}, \alpha_{0}=0$ ?' and Fig. 5 is the answer given by eq. (4) for the usual spectrum and deep water. Here too the wall is not shown, but it is easily recognizable because clearly along its line the water surface is discontinuous. 
We see a wave group that approaches the wall and its front center targets on the wall-end. Thus one half of the wave font goes beyond the breakwater and penetrates into the protected area, and the other half impacts the wall and is raised because of reflection. In line with the above put question, at time instant to $\left(0\right.$ in the picture) at given point ro, $\alpha_{0}$ there is the crest of the wave of given very large height $H$. It is the first diffracted wave crest, beyond the wall-end.

The given very large wave height $H$ in the figures of this paper is thought of as the maximum expected wave helght during a sea state at given point xo. yo. For this reason wave height $H$ in Fig. 5 is smaller than in the foregoing figures.

\section{EXPERIMENTS AT SEA TO VERIFY THE THEORY}

\subsection{Site, facilities, instruments and sea states}

A laboratory for maritime engineering has been prepared starting on 1989 at the Reggio Calabria beach. The beach faces Messina Straits, a clear sea where tide has a maximum excursion within.3.m. The wind blows very often from the NW, that is from Sicily, and after a few days of NW winds the southerly swells disappear, and the sea states consist of pure wind waves. The NW fetch is of about $10 \mathrm{~km}$ (see Fig.6) and the significant height and the dominant period of the spectrum are typically 0,15 à $0,35 \mathrm{~m}$ and 2 à $2,5 \mathrm{~s}$.

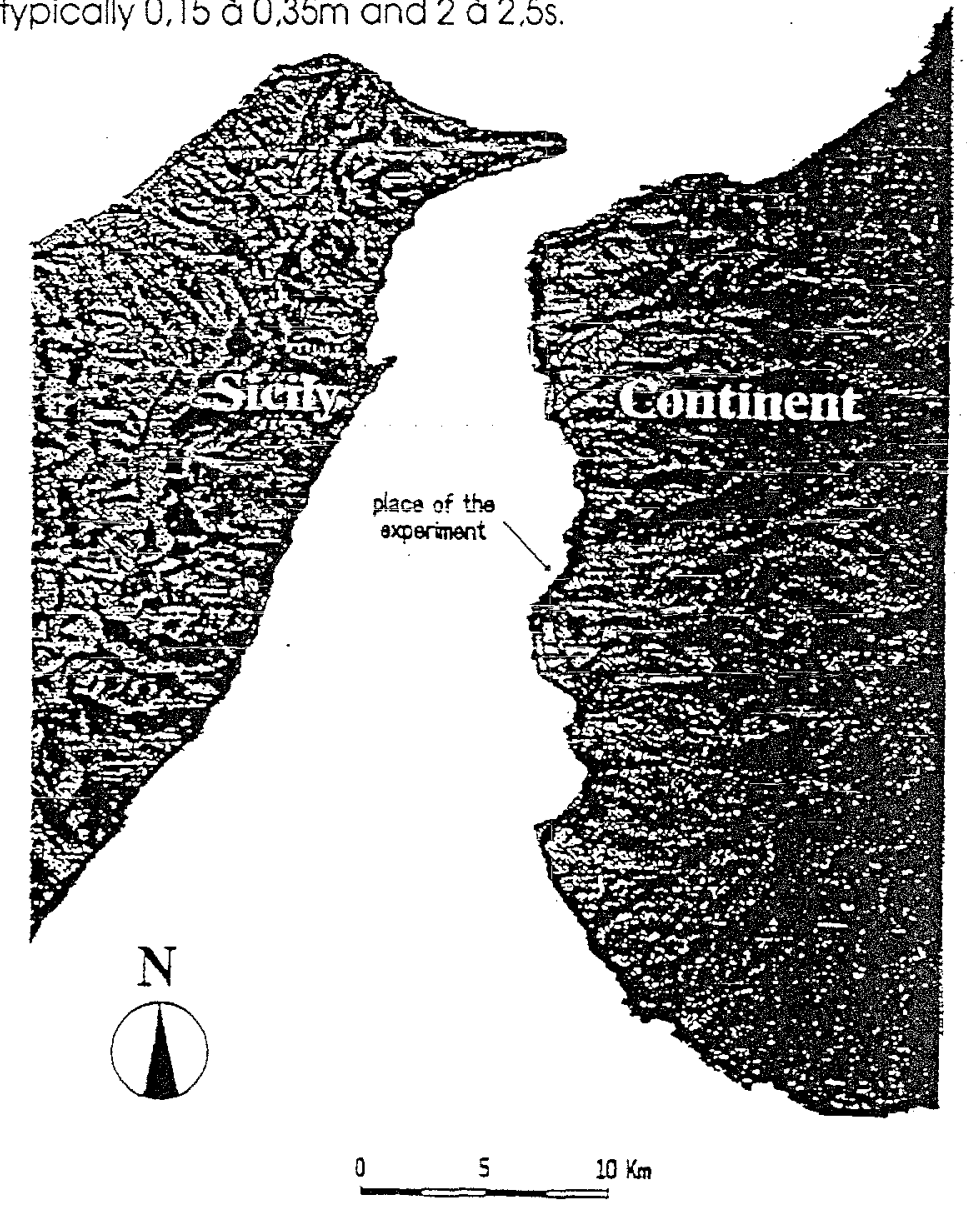

Fig.6: The site of the experiment 
The laboratory occupies about $60 \mathrm{~m}$ of littoral and is equipped with small buildings. One of the buildings keeps the electronic station which is connected by submarine cables to the gauges at sea.

A first experiment was executed on may 1990 in order to verify the quasideterminism theroy for what concerns the progressive waves. To that end, 9 piles each supporting 2 wave meters and a pressure transducer were placed at sea. The disposition of the piles was designed to intercept the wave group and follow part of their course. The results are the object of a paper which is presently under review for publication, and they will be also the object of a lecture to the 23rd Congress on Coastal Engineering.

Here, in what follows a first news is given of a second experiment which was executed on may 1991. The experiment dealed with the second part of the theory, that is: waves interacting with reflecting walls.

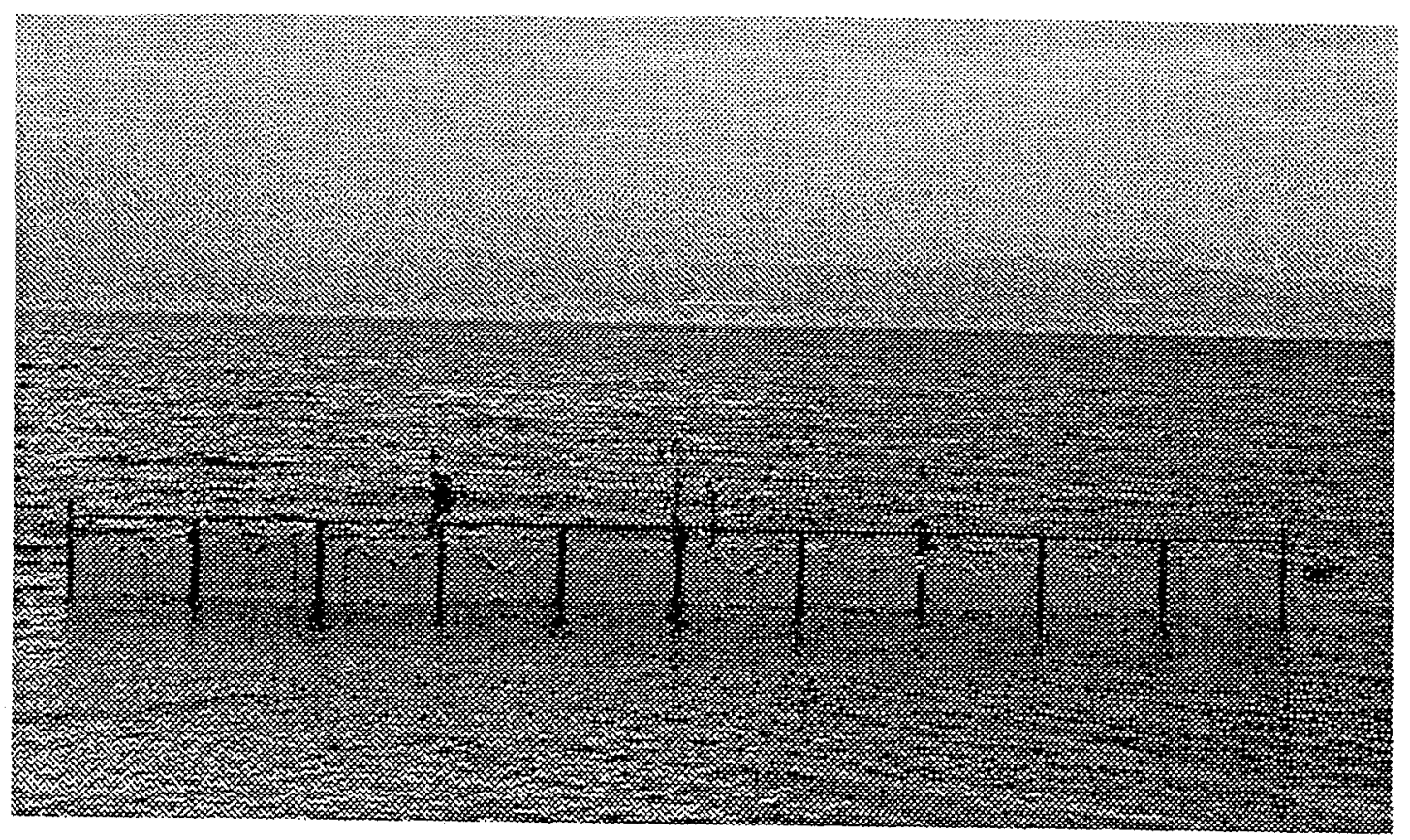

Fig. 7 : The reflecting wall in a photo from the beach

A special vertical reflecting wall $12 \mathrm{~m}$ long $\times 2.1 \mathrm{~m}$ high was built on a rubble mound: the water depth at the wall basis was $1.5 \mathrm{~m}$ and the maximum vertical thickness of the rubble mound was $.25 \mathrm{~m}$. The structure consisted of a steel truss whose stability was ensured by a dead weight of pig iron disks. The bearing slab was provided with teeth so as to improve the friction with the rubble mound. The reflecting vertical plane of $12 \mathrm{~m} \times 2.1 \mathrm{~m}$ was formed by alluminium panels which were fixed to the truss.

Before (seaward) the wall 3 horizontal beams supported 30 pressure transducers. Both the horizontal beams and the piles were designed to get an high degree of stiffness as well as a small section, and in particular each vertical pile was stiffened by 4 steel cables and its diameter tapered from 0.07 to $.04 \mathrm{~m}$. The bottom depth in the area covered by the instruments was on the average $.5 \mathrm{~m}$ greater than at the wall, that is, it was $2.0 \mathrm{~m}$ with respect to the M.W.L. 
The electronic station at the present is able to sample data only from 16 gauges. Therefore 3 teams of gauges were formed from the 30 pressure transducers. Each team had to carry out a new experiment. In particular experiment number 2 had to verify the predictions on how a very high wave forms at the wall and how it does at some distance from the wall : 9 gauges along the wall and 7 gauges on a horizontal wall-parallel beam were connected in order to record the wave front and to estimate the path of the front center - see Fig.8-.

In all. 52 sea states consisting of pure wind waves (with a negligible swell component) from the NW fetch, were recorded. Each sea state was of 9 min which correspond to a number of waves ranging from 230 to 280 .

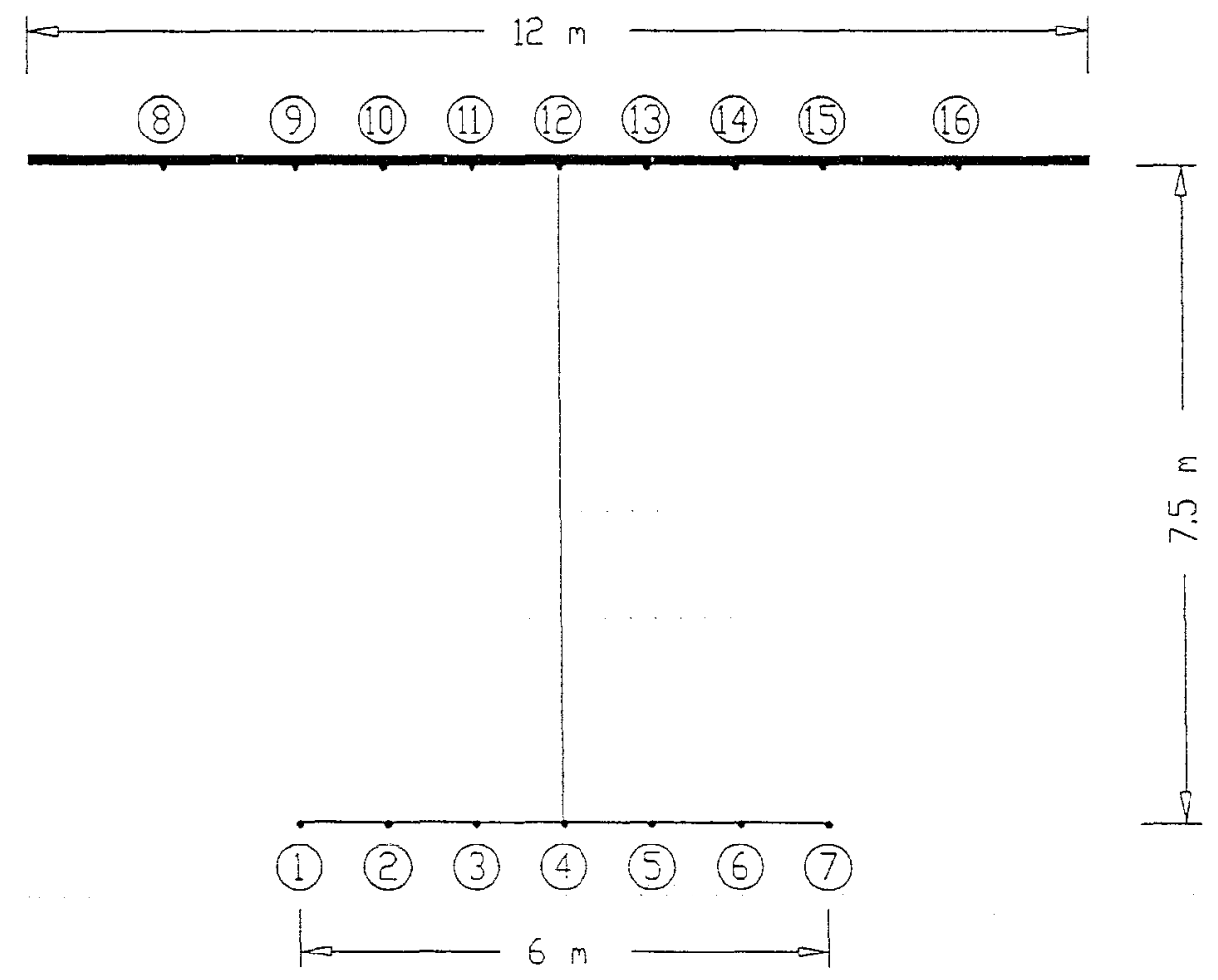

Fig. 8: Plan of the gauges in the experiment on how a very high wave forms at the wall.

\subsection{Deterministic waves from the measured random waves of the pressure} head

To Stoke's first order, elevation $\xi_{R}$ of the pressure head waves, being proportional to the time derivative of stationary Gaussian random process $\phi_{R}$ is in its turn a stationary Gaussian process of time at any point. Therefore result (4) of sect. 2 holds also for the pressure head waves, that is : given a wave of the 
pressure head with a very large height $H \quad\left(\frac{H}{\sqrt{m_{00}}} \rightarrow \infty\right)$ at a given point $x_{0}, y_{0}, z_{0}$, the probability approaches 1 that the elevation of the pressure head waves approaches the deterministic function

$$
\begin{aligned}
& \xi_{D}\left(x_{0}+X, y_{0}+Y, z_{0}+Z, t_{0}+D\right)= \\
& =\frac{H}{2}\left[<\xi_{R}\left(x_{0}, y_{0}, z_{0}, t\right) \xi_{R}\left(x_{0}+X, y_{0}+Y, z_{0}+Z, t+D\right)>+\right. \\
& \left.\cdot<\xi_{R}\left(x_{0}, y_{0}, z_{0}, t+T^{*}\right) \xi_{R}\left(x_{0}+X, y_{0}+Y, z_{0}+Z, t+D\right)>\right] / \\
& I\left[<\xi_{R}^{2}\left(x_{0}, y_{0}, z_{0}, t\right)>-\left\langle\xi_{R}\left(x_{0}, y_{0}, z_{0}, t\right) \xi_{R}\left(x_{0}, y_{0}, z_{0}, t+T^{*}\right)>\right]\right.
\end{aligned}
$$

where to is the instant of the crest of the pressure head wave of given height $\mathrm{H}$ at given point $x_{0}, y_{0}, z_{0}$ and $T^{*}$ is the abscissa of the absolute minimum of the autocovariance

$$
\psi_{P}(D)=\left\langle\xi_{R}\left(x_{0}, y_{0}, z_{0}, t\right) \xi_{R}\left(x_{0}, y_{0}, z_{0}, t+T\right)\right\rangle .
$$

Here the subscript $p$ has been used to distinguish the pressure head waves from the surface waves.

Deterministic elevation $\xi_{D}$ can be quite easily calculated from the time series data of $\xi_{R}$. In particular let $x_{0}, y_{0}, z_{0}$, be the coordinates of the ith gauge and $x_{0}+X$. $y_{0}+Y, z_{0}+Z$ the coordinates of the $j$ th gauge, then deterministic elevation $\xi_{0}$ at point $j$ is

$$
\xi_{\mathrm{Di}}\left(t_{0}+\mathrm{T}\right)=\frac{H^{2}}{2} \frac{\left\langle\xi_{R i}(t) \xi_{R i}(t+T)>-<\xi_{R i}\left(t+T^{*}\right) \xi_{R i}(t+T)>\right.}{<\xi_{R i}^{2}(t)>-<\xi_{R i}(t) \xi_{R i}\left(t+T^{*}\right)>}
$$

where to is the time instant of the crest of the pressure head wave of given height $H$ at point 1 and $T^{*}$ is the abscissa of the minimum of autocovariance $\psi p(T)$ at point i

$$
\Psi_{P}\left(D=\left\langle\xi_{R i}(\dagger) \cdot \xi_{R i}(\dagger+T)\right\rangle\right.
$$

\subsection{How a very high wave forms at the wall: experimental verification}

Provided to know that at the wall center at a time instant to there is a wave with a height $H$ very large with respect to the mean, what can we expect to happen before and after to at the 16 points of experiment 2 ?. An answer is given in Fig. 9 that has been calculated by means of eq.(10) from the pressure head measured during sea state 6 ; that is, eq. (10) has been applied in the form (12). and gauge $i$ of the given very large wave height $H$ is gauge 12 at the wall center. We would remark that in Fig. 9 have not been artificially smoothed: simply the time series data of pressure head $\xi_{R}$ sampled during 9 consecutive minutes has been inserted in eq.(12) and what is shown by the pictures is the output. 

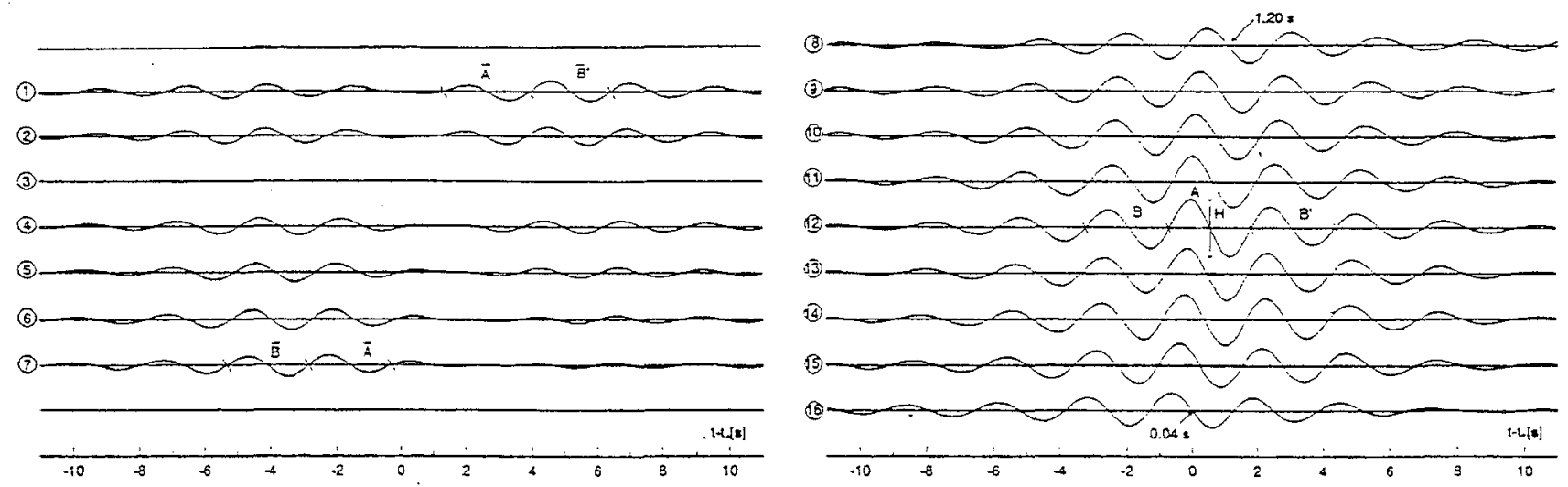

Fig.9: This picture was obtained by applying eq.(10) with the time series data of pressure head $\xi_{R}$ of sea state 6, without any artificial smoothing. The point of gauge 12 has been taken as $x_{0}$, yo so that, according to the quasi-determinism theory, the figure shows what we expect to happen at the 16 points in the case that a wave with a very large height $H$ should be recorded at point 12 at the wallcenter

The wave of the given very large height $H$ occupies the center of a group. and is marked by letter A. We see that the wave attacks the wall from the right (for a people looking at the wall from seaward). Indeed the wave is recorded first by gauge 16, then by gauges 15,14 and in the end by gauge 8 . The time shift of wave A at two different points proves to be proportional to the distance between the two points. This observation reveals that the central wave front of the group is straight, like in the theoretical wave groups of the 3-D pictures. The angle between the wave front and the wall is $\left|\theta_{0}\right|=\arcsin \left(c^{*} \frac{D t}{D x}\right)$ where c is the wave celerity. Dt is the time shift (absolute value) of the wave record at two points, and $D x$ is the distance between the two points along the wall-axis. In particular, Fig. 9 shows that the time shift from gauge 16 to gauge 8 is Dt $=1.20-0.04=1.16 \mathrm{~s}$. Then, the distance between the two points is $D x=9 \mathrm{~m}$ and the wave celerity is $c-3.5 \mathrm{~m} / \mathrm{s}$ $\left(T_{d}=2.50 \mathrm{~s}, h=2.10 \mathrm{~m}\right)$ so that $\left|\theta_{0}\right|$ turns out to $b e 25^{\circ}$, and the angle is negative because the attack is from the right.

Now if the reflection is mirrorwise, the center of the wave front has to describe a trajectory like that of Fig. 10: when approaching the wall, it should pass at gauge 7 and when going back seaward it should pass at gauge 1 . 
SESSION 1 - Houle, marée el surcote, modélisation

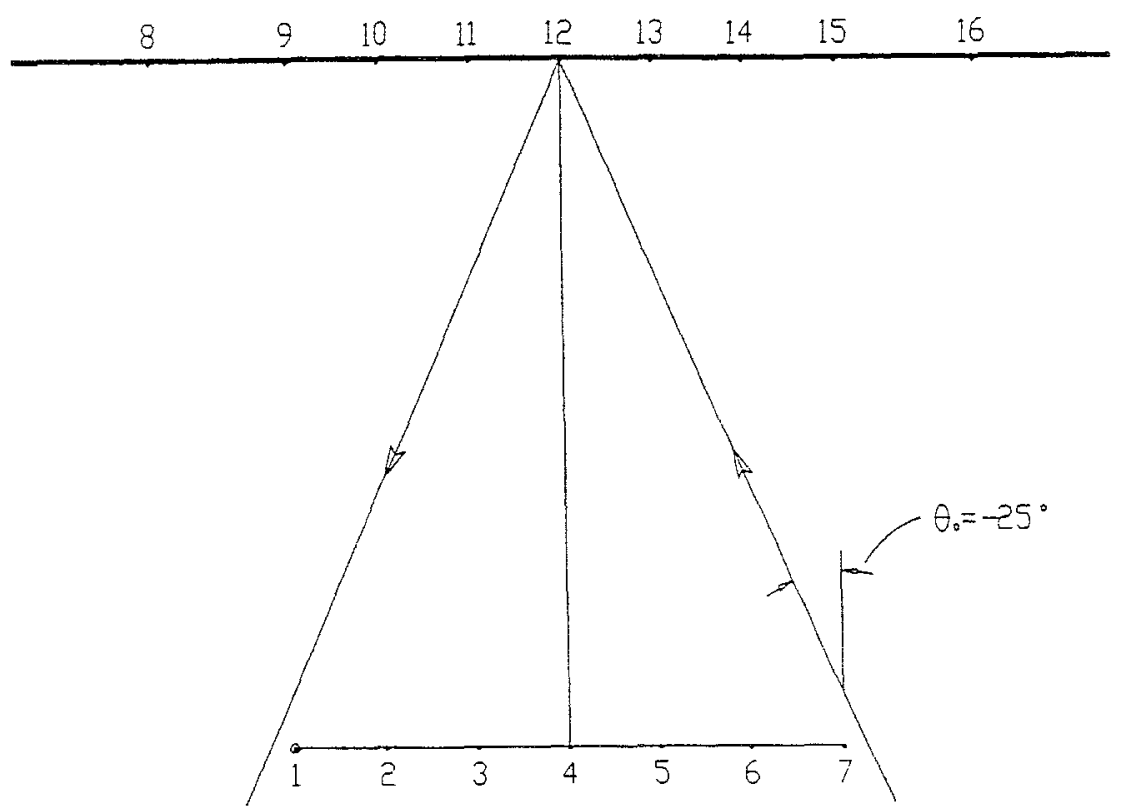

Fig. 10: The trajectory of the center of the group of Fig. 9

An apparent confirmation comes from the records of the 7 seaward gauges 1 - 7: before instant to the wave front transits at the 7 gauges and the maximum height is at point 7 , after to the wave front transits at the 7 gauges and the maximum height is now at point 1 .

We can also note that at point 7 the envelope center is within wave $B$ and wave A. After a course of about one wave length, at point 12. wave B passes into the envelope head and wave $A$ passes to occupy exactly the envelope center.

Finally, at point 1, after a course of one more wave length, the envelope center is within wave $A$ and following wave B'. Thus we get also confirmation that the waves alternate themselves at the envelope center. It is the basic phenomenon of the wave group mechanics, which can be appreciated if the marked wave of 3-D Fig. 1 is followed during its course.

\subsection{What happened when the highest wave of the experiment was recorded and what had been predicted}

The highest wave of the whole experiment (it is meant 'the highest wave with respect to the root mean square wave elevation') was recorded by gauge I during sea state 51. The wave had a crest-to-trough height of $9.6 \sqrt{\mathrm{mo}_{0}}$ that is nearly 2.5 times the significant height of its sea state.

Fig. 11 (upper panel) shows the record made by gauge $I$ and by the other gauges. A few instruments were out of order because they had been damaged by a storm. 

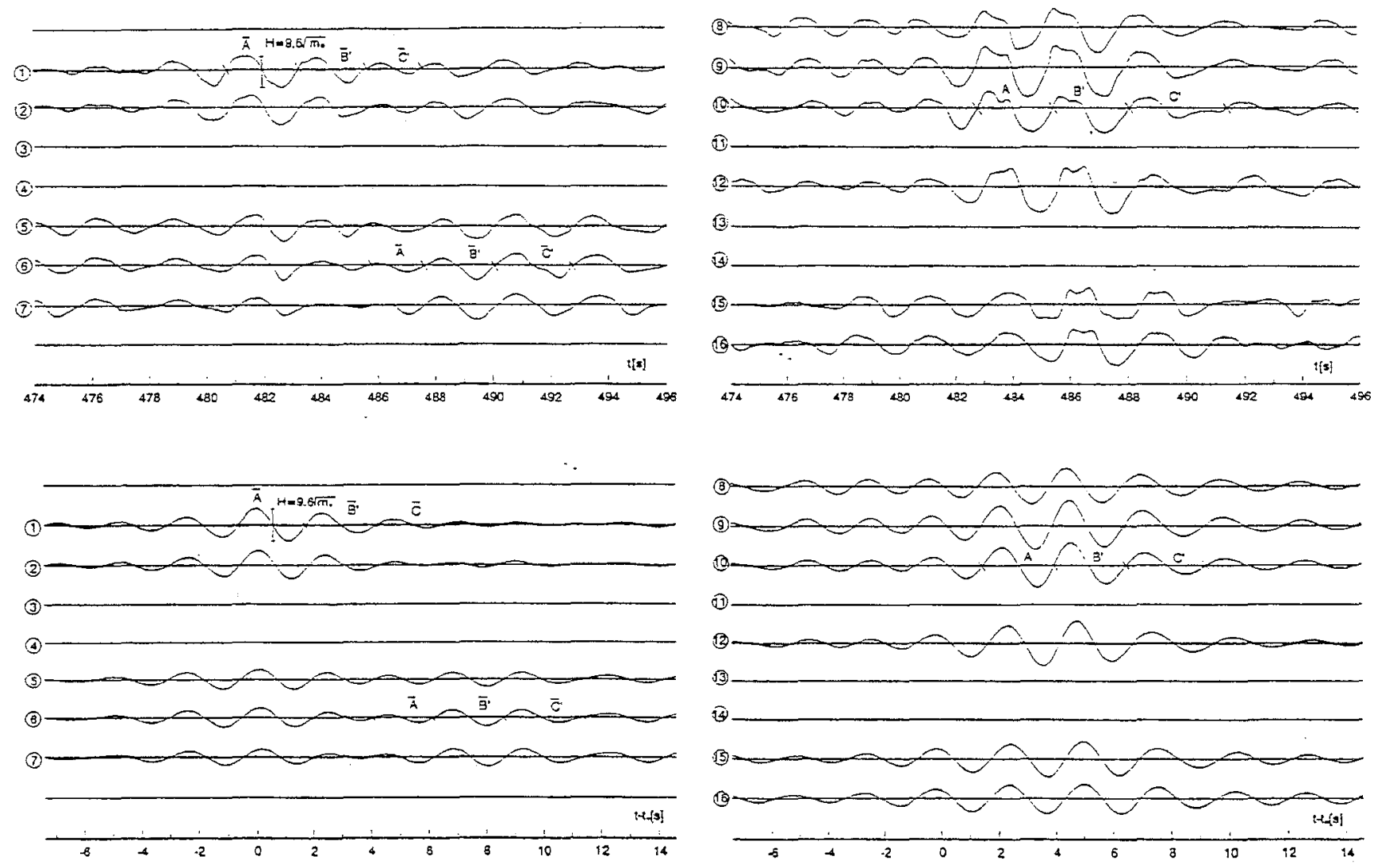

Fig. 11 : Upper panel : record of the group with the largest wave height of the whole experiment. Lower panel, : prediction by means of eq. (10) with the time series data, without any artificial smoothing.

Fig. 11 (lower panel) shows what is predicted by the quasi-determinism theory. The calculation was made by means of eq. (12) with the time series data of pressure head $\xi_{R}$, without smoothing.

We see that the quasi-determinism theory foresee what essentially happens. Both the prediction and the wave record show first a wave group that transits at the 7 seaward gauges (gauges $I$ - 7 ) with large height $H$ at the group's center. The group is approaching the wall from the left (for a people looking at the wall from seaward), and indeed we see that wave front $A$ at the envelope center first is recorded by gauge $I$ and in the end by gauge 1 . Angle $\theta_{0}$ estimated from the time shift of wave $A$ on the 7 seawara gauges proves to be of about $15^{\circ}$ both in the prediction and in the recorded wave group.

Thus, from the time shift, at gauges 1 - $\underline{Z}$, of the group approaching the wall, we expect that the front center strikes somewhat on the left of the wall-center (cf. the trajectory of the group in Fig. 12), and this is consistent with what we see in Fig. 11 at the 9 points along the wall.

Finally, the wave group, after having been reflected transits one again at the 7 seaward gauges and, this time, the front center passes on the right side. that is, on gauges $6, Z$. 


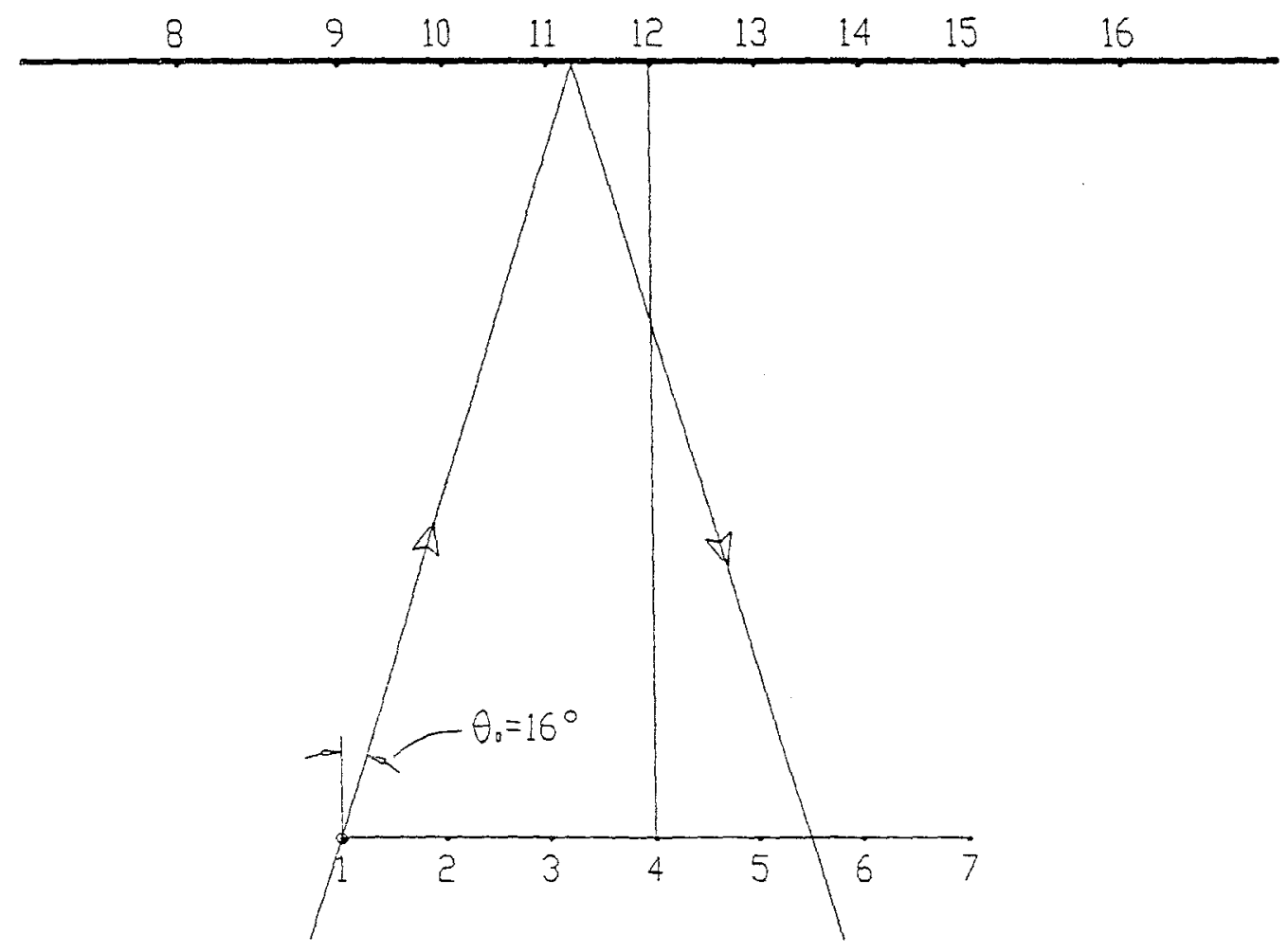

Fig. $12:$ The trajectory of the center of the group of Fig. 11

Also the alternation of the waves at the envelope center is apparent both in the prediction and in the record. When the group transits for the first time at the 7 seaward gauges, wave A occupies the envelope center. Then, at the wall. after a course of about 1 wave length, the envelope center falls within wave $A$ and wave $B^{\prime}$, that is, wave $A$ has passed into the group head, and wave $B$ ' is going to occupy the envelope center. Finally, when the group passes once again at gauges $I-I$. wave $B$ in its turns is leaving the envelope center which is going to be occupied by succeeding wave $C$. 


\section{REFERENCES BIBLIOGRAPHIQUES}

(1) Bocotti P., Sea waves and quasi-determinism of rare events in random processes, Atti Acc. Naz. Lincei, Rendiconti, 76, 2, 1984.

(2) Boccotti P., Refraction, reflection and diffraction of irregular gravity waves, in Excerpta of the Italian Contribution to the field of hydraulic engineering, vol.3, 47-89, Libreria Proetto Padova Publ., 1988.

(3) Boccotti P. On mechanics of irregular gravity waves, Atti Acc. Naz. Lincei, Memorie. 19.5, 111-170, 1989.

(4) Forristall G.Z. et al., Directional wave spectra in hurricane Carmen and Eloise, Proc. Conf. on Coastal Engng. ASCE, 1980.

(5) Hasselmann $K$ ef al. Measurements of wind growth and swell decay during the North Sea Wave Project (JONSWAP), Deut. Hydrogr. Zeit., A-8. 1973.

(6) Longuet-Higgins M.S., the effects of non linearities on statistical distributions in the theory of sea waves, J. Fluid Mech., 17, 1963

(7) Penny W.G. and Price A.T., The diffraction of sea waves by breakwater. Phil. Trans. Roy. Soc, A-244, 1952.

(8) Phillips O.M. The theory of wind generated waves, Adv. in Hydroscience, 4. 119-149, 1967. 